Revista de la red interuniversitaria de estudios sobre las literaturas rioplatenses contemporáneas en Francia

Hors-série | 2019

Ricardo Piglia: Cierta idea de literatura

\title{
Los diarios de Piglia: Recuperación de la gestación de Plata quemada
}

\section{Daniel Balderston}

\section{OpenEdition Journals}

Edición electrónica

URL: http://journals.openedition.org/lirico/7941

DOI: $10.4000 /$ lirico.7941

ISSN: 2262-8339

Editor

Réseau interuniversitaire d'étude des littératures contemporaines du Río de la Plata

\section{REFERENCIA ELECTRÓNICA}

Daniel Balderston, « Los diarios de Piglia: Recuperación de la gestación de Plata quemada ", Cuadernos LIRICO [En línea], Hors-série | 2019, Puesto en línea el 27 febrero 2019, consultado el 10 mayo 2019. URL : http://journals.openedition.org/lirico/7941 ; DOI : 10.4000/lirico.7941

Este documento fue generado automáticamente el 10 mayo 2019

Cuadernos LIRICO está distribuido bajo una Licencia Creative Commons Atribución-NoComercialSinDerivar 4.0 Internacional. 


\title{
Los diarios de Piglia: Recuperación de la gestación de Plata quemada ${ }^{1}$
}

\author{
Daniel Balderston
}

Para John Kraniauskas

1 Uno de los placeres de leer Los diarios de Emilio Renzi de Ricardo Piglia es la posibilidad de observar el surgimiento de algunos de los proyectos del escritor. Los diarios publicados no son más que una pequeña muestra de los cientos de cuadernos de Piglia (los cuales fueron tema de un lúcido documental de Andrés Di Tella), y es notable que el escritor, aun sufriendo una inmovilización progresiva a causa de la esclerosis lateral amiotrófica (ELA), y asistido durante esos años tanto por ayudantes como por una computadora diseñada especialmente para él, haya sido partícipe en la selección de tal publicación con la evidente intención de proveer a sus lectores de una mejor comprensión de las etapas temprana e intermedia de su gestión escritural. El primer volumen, titulado Años de formación y publicado en 2015, cubre el período desde 1957 (cuando Piglia aún era adolescente) hasta 1967 (año en que publica La invasión, su primer libro de cuentos). El segundo volumen, Los años felices, publicado en 2016, va de 1968 a 1975, cuando Piglia estaba a punto de publicar su segundo libro, Nombre falso, que incluye el magistral "Homenaje a Roberto Arlt". El tercer volumen, Un día en la vida, apareció en 2017, y actualmente se están procesando, según se dice, varias otras publicaciones de selecciones del diario.

2 La novela Plata quemada salió en 1997 y ese mismo año ganó el Premio Planeta (premio que fue retirado a causa de pleitos, aunque veinte años después pienso que esta magnífica novela merecía ese galardón). La novela trata del robo de un camión blindado en 1965 en San Fernando, uno de los suburbios de Buenos Aires, y la huida de algunos de los criminales a Montevideo, donde, en medio de un intercambio a tiros con la policía, tres de ellos fueron rodeados. Siempre se supo que la novela estaba basada en hechos reales, pero la magnitud de la investigación de Piglia no estuvo clara hasta la publicación de los diarios. En este artículo examinaré la relación entre el joven escritor de un diario y el autor maduro que publicó una novela basada en las indagaciones consignadas en algunas de esas entradas treinta años después. Debo añadir que el periodista uruguayo Leonardo 
Haberkorn ha investigado ampliamente el crimen y los criminales, y ha publicado tanto un libro, titulado Liberaij: La verdadera historia del caso Plata quemada (2015), como una cantidad considerable de material sobre el caso, al que se puede acceder en la web. Este artículo se concentrará más en el diario de Piglia que en las fuentes consultadas por Haberkorn. Casi toda la fase de preparación de Plata quemada está en el primer volumen de los diarios, que acaba en 1967; la publicación tuvo lugar tres décadas después. Esta prolongada e inusual gestación es la que me propongo considerar. Abordajes anteriores han situado la novela entre el género del suspenso y el policial (Rodríguez Pérsico 2004), han comentado la "forma cuasi periodística" de la novela (Clayton 2004: 136), y se han preguntado sobre la investigación de los hechos en el texto (Premat 2004); este artículo es, me parece, el primero que toma en consideración los diarios de Piglia.

El robo del camión blindado ocurrió en San Fernando el 28 de septiembre de 1965. El 5 de noviembre más de trescientos cincuenta policías rodearon a los tres criminales que sobrevivieron al episodio del robo. Estos eran Marcelo Brignone, Carlos Alberto Mereles y Roberto Dorda, quienes se refugiaron temporariamente en el Edificio Liberaij en Montevideo, en Julio Herrera y Obes, entre Canelones y Maldonado, y, de acuerdo a Piglia, después de 16 horas de balacera, el sobreviviente, Dorda, fue arrestado y posteriormente extraditado a Buenos Aires (donde fue asesinado un año después, en prisión) ${ }^{2}$. El martes 9 de noviembre, cuatro días después, Piglia escribe en su diario: "Acaso el final de mi novela sobre Cacho está en el departamento de Montevideo en esos tres pistoleros atrapados allí, aguantando dieciséis horas y resistiendo contra cuatrocientos policías y soportando gases, fuego, balas, agua, bombas hasta que al fin queman el dinero y gritan: "Vengan a buscarnos, guanacos"”' (I, 203) 3 . Esta es la primera de las muchas entradas de Piglia que siguen el caso ${ }^{4}$.

4 A lo largo de 1966 y 1967 hay entradas frecuentes relacionadas con las investigaciones de Piglia y sus intentos de escribir una novela al respecto. El proyecto de escribir una novela sobre el "camión pagador", estaba entrelazado a la intención de Piglia de escribir una novela sobre Cacho, un amigo cercano suyo que era ladrón; buena parte del primer volumen de los diarios publicados se enfocan no tanto en las aventuras de Cacho, sino en la manera en que éste las narraba, cosa que fascinaba al joven Piglia. Así, por ejemplo, el 27 de enero de 1966 Piglia escribe:

Quizá en la novela pueda construir a Cacho a partir de mi propia adolescencia; darle a él la experiencia de mi vida en esos años, extraerla de mis diarios. Además es necesario encontrar una anécdota casi policial (y que todos ya conozcan y no haya que explicar, como sucede con los hechos de la tragedia griega: todos conocían los mitos y el argumento en el que se basaban las obras). Por ejemplo, el robo al camión pagador y la fuga a Uruguay de los maleantes. Usar entonces el pretexto de la no ficción para escaparle al verosímil y al costumbrismo. Escribir, digamos así, un drama épico, o mejor, una tragedia. (I, 221)

El prólogo y los ensayos cortos de Piglia sobre escritores estadounidenses, publicados en diciembre de 2016 en Escritores norteamericanos, aunque escritos por primera vez en 1967, confirman su fascinación por "la no ficción", género que estaba siendo cultivado en Argentina por su amigo Rodolfo Walsh (quien aparece frecuentemente en los dos primeros volúmenes del diario). Es interesante observar cómo Piglia intenta combinar tres elementos distintos en la novela: sus experiencias de adolescente (la mudanza de Adrogué a Mar del Plata por los líos políticos de su padre, sus estudios en La Plata, la escritura de sus primeros cuentos) con la experiencia de Cacho como ladrón, y con el robo y huida de los criminales del camión blindado: trama esta última que está tan fresca en su 
memoria cuando escribe las entradas, que parecería innecesario tener que explicarlas (cosa que cambia cuando publica la novela treinta años después, como él mismo comenta en el epílogo a Plata quemada).

6 La próxima entrada sobre este proyecto en el diario publicado se escribe nueve meses después, el 12 de octubre de 1966. Aquí Piglia está corrigiendo su primer libro de cuentos (publicado como Jaulario en Cuba y La invasión en Argentina), y pensando en un nuevo proyecto:

Me resisto a corregir los cuentos, tengo la certidumbre de que están bien escritos en la primera redacción. Como si el estilo se fijara de una vez y cualquier "mejora" (convencional) destruyera el efecto del conjunto. Igual tengo que cerrar el libro y olvidarme de él para poder empezar a escribir la historia de los maleantes que huyen a Uruguay. Tan cercana a mí que necesito escribirla como si estuviera sucediendo ahora. (I, 261)

El nombre asociado al proyecto cambió de "el camión pagador" a "los maleantes que huyen a Uruguay". Además, la cercanía de Piglia a los hechos sigue siendo intensa. Fue en este período que indagó e hizo entrevistas sobre el caso, si es que le creemos al epílogo de la novela, cosa que explicaría tal cercanía.

8 En la primera entrada de 1967, fechada el 2 de enero, Piglia escribe: "Hoy empecé las notas preliminares para la novela de los malandras que escapan a Montevideo" (I, 281) ${ }^{5}$. Dos días después, escribe:

Anoche trabajé hasta las tres de la mañana anotando algunas situaciones. Alguien entrega el golpe al banco de San Fernando. Establece un contrato por el que, a cambio de la información, recibirá una parte del dinero robado. Esa situación no va a ser contada directamente, sólo se ven los efectos. (I, 282)

Esta entrada muestra que Piglia pasó del proceso de recolección de información, y la reflexión sobre ella, a la forma literaria que quiere en su novela. Treinta años después la novela integrará esa manera oblicua de narrar, a pesar de que la forma final, una narración en tercera persona con cambios graduales en la focalización de personaje a personaje, va más allá del horizonte del Piglia de los diarios. Las dos entradas siguientes tratan de On Murder Considered as one of the Fine Arts de De Quincey, In Cold Blood de Capote (ambos textos tratan sobre cómo narrar un crimen) y Los hijos de Sánchez de Oscar Lewis (texto clave en el desarrollo del género del testimonio, pero que no se asocia a la ficción policial como los otros dos). El 10 de enero Piglia escribe: "La anécdota central de la novela es la encerrona, pasan tres días sin salir del departamento en Montevideo. De pronto llega la policía y empieza el asedio y la batalla que dura toda la noche" (I, 285). Una semana después, el 17 de enero, escribe:

La novela. El pistolero y la muchacha, una historia de amor. Pero atrás, en secreto, ella se acostaba con otros empujada por él. En el final ella muere con un tiro en la espalda. Casi por casualidad, una bala que rebota y la mata en el baño. El amor, entonces, romántico, y a la vez, por supuesto, como siempre muy perverso, entre el Inglés y Moira, la muerte de ella al final, cuando casi se odian y sólo queda el remordimiento. (I, 287)

Esta nota muestra la brecha entre el proyecto inicial y la novela publicada treinta años después, que, los lectores recordarán, enfoca otro tipo de relación romántica, la relación homosexual entre Dorda y Brignone. Como discutí hace algunos años en un artículo sobre la novela, esa relación está extrañamente mediada por referencias al Martín Fierro de Hernández, que se toma (tal vez por la lectura que hace del poema Martínez Estrada) como el texto primario del homoerotismo literario argentino, nada de lo cual está 
presente aquí. Habenkorn cita al juez Molteri, que falló contra la hija de Dorda en el caso sobre la novela de Piglia:

Comprendo que no deja de ser desagradable que de un ascendiente se diga innecesariamente que era homosexual y drogadicto, como que se relaten numerosos episodios donde el personaje desempeña descaradamente esos roles. Pero cuando ese mismo protagonista desarrolló conductas reales de maleante y frío asesino, esos aspectos de su vida, en rigor, resultan desproporcionadamente minúsculos y hasta anecdóticos, como para agravar el sentimiento de deshonra o la carga infamante que debieron sentir sus descendientes por la desdorosa conducta de aquél. (Habenkorn 2015: 212)

11 Es interesante observar cómo el juez distingue entre distintos tipos de infamia. También interesa el hecho de que Piglia decide en este asunto, como en "La invasión”, enfatizar la homosexualidad 6 .

El 17 de abril de 1967 Piglia registra que ha rentado un cuarto lejos del centro de Buenos Aires, para concentrarse en la escritura de la novela, esto gracias a la generosidad de su editor Jorge Álvarez, quien, entre otras cosas, le pagaba para que compilara una serie de antologías (y algunos prólogos de los recogidos luego en Escritores norteamericanos): "Asegurado económicamente, instalado ya en un lugar en el sur de la ciudad, puedo empezar por fin a escribir la novela sobre el robo al camión pagador en San Fernando" (I, 305). Unos cuantos meses después, el 17 de septiembre de 1967, escribe otra vez sobre la novela, ahora con un título tentativo:

El robo es, digamos, una historia sucia, porque en la fuga todo se altera. Narrada en plural, en coro, pero sin nadie que decida sobre el sentido de los hechos. Ellos planean el atraco al camión pagador en complicidad con la policía y luego se escapan rompiendo el pacto. (I, 326)

Poco menos de dos semanas después, el 29 de septiembre, escribe: "La novela del atraco se va a llamar Entre hombres" (I, 329). Lo que sugiere la posibilidad de que Piglia esté pensando en términos del romance homosexual como un elemento importante en la novela publicada, idea que ya había sido tema en el cuento "La invasión", cuya acción ocurre en una celda, relato que le dio el título a la edición argentina del libro que acababa de publicar.

14 El 12 de diciembre, en la más prolongada de sus entradas en el diario, Piglia escribe:

Entre hombres. Éstos son los primeros resultados de una investigación más vasta que he comenzado a partir de las experiencias de Oscar Lewis (Los hijos de Sánchez, La vida). Como se sabe, Lewis ha renovado el campo de la antropología a partir del empleo del grabador de cinta con la intención de grabar historias de vida, hechos reales, $y$, a la vez, narrados con la voz y el estilo de los protagonistas. Me interesa sobre todo plantear aquí algunas consideraciones. Como se sabe, el uso del grabador modifica el nivel de las exploraciones de la experiencia y genera una distancia con el que está contando los acontecimientos. Tratamos [¿Tratando?] de fortalecer ese criterio, yo he reconstruido en este libro los hechos que han sucedido a partir del asalto al camión pagador de un banco de San Fernando, y la posterior huida a Montevideo de los maleantes, que fueron por fin rodeados por la policía en una encerrona producida por una delación. Mi primer contacto con los protagonistas de esta historia sucedió el 11 de enero de 1965, al leer en el diario La Razón la noticia del asalto. A partir de ahí entrevisté a todos los testigos y participantes en los acontecimientos y pude acceder a las grabaciones realizadas por un radioaficionado de las conversaciones que mantuvieron los malvivientes mientras resistían el ataque de la policía. No hace falta que fatigue al lector con la narración de las dificultades que tuve que afrontar para realizar las grabaciones y lograr un acercamiento personal con los protagonistas. Fueron necesarias varias entrevistas 
para lograr conocer con algún detalle los hechos. Por fin conseguí unirme a un grupo de cinco testigos con los que inicié las primeras conversaciones sin grabador, de esta manera establecí con ellos una relación de confianza, y cuando por fin comenzamos a trabajar con el registro de su relato ya estábamos en una relación muy fluida. (I, 332-333)

15 Esta entrada tiene más o menos el mismo tono del epílogo a la novela de 1997: se concentra tanto en la investigación de Piglia sobre el caso, mencionando haber incluido entrevistas con varios testigos y protagonistas que proceden de ella (incluyendo Dorda, según comenta en el epílogo de la novela [1997: 247]), como también en sus preocupaciones sobre cómo acortar la distancia entre él y quienes entrevistó, distancia que es efecto colateral de la dinámica de las grabaciones mismas. Lo que revela la entrada de 1967, que no se encuentra en el epílogo de 1997, es la importancia que tenía el antropólogo Oscar Lewis para el joven Piglia: específicamente la manera en que el antropólogo, en el trabajo de campo en el que empleó la grabadora como herramienta de investigación, logró difuminarse a sí mismo en la narración para permitirle a sus entrevistados hablar con voz propia. Lewis -y Capote, quien negó haber empleado una grabadora, y ni siquiera un cuaderno, en las entrevistas que culminaron en In Cold Bloodes a quien se recurre como modelo sobre cómo narrar la historia. Este modelo es descartado en la novela publicada, pero saber que Piglia inicialmente pensó en cómo permitirle a la novela fluir "con la voz y el estilo de los protagonistas" es clave.

16 Ésa es la última entrada sobre el proyecto que culminó en Plata quemada, dentro del primer volumen (1957-1967) del diario publicado. Cinco años después Piglia se mantiene interesado en el caso y sus notas del 14 de junio de 1970 revelan que continúa trabajando en la novela:

Sigo trabajando una novela que interiormente considero frustrada, la cuento aquí para que quede algo de todos esos meses de trabajo: un relato de cien páginas basado en el robo a un camión pagador en San Fernando, que está centrado en el encierro y el acoso al que son sometidos los maleantes, que resisten en un departamento asediado por la policía, en Montevideo. Escrito en tercera persona clásica, combina la situación de clausura (resisten toda la noche y mueren al alba luego de quemar la plata) con la biografía del rehén a quien capturan al entrar al edificio. Toda la novela respeta la unidad de tiempo y de lugar y está cruzada por el monólogo del que ha sido capturado (un jugador de fútbol uruguayo). (II, 193-194)

17 Algunos de los comentarios sobre la escritura de la novela sobre el caso Liberaij están intercalados con notas de lo que acabó siendo "Homenaje a Roberto Arlt" y Respiración a rtificial. Obviamente, el proyecto se dejó a un lado y se retomó muchos años después, luego de La ciudad ausente (1992) y de los relatos de Prisión perpetua (1988).

18 El tercer volumen, que va de 1976 a 1982 e inserta algunos escritos adicionales desde entonces hasta la muerte de Piglia, contiene sólo tres notas sobre Plata quemada. El 25 de junio de 1979, Piglia escribe:

He tratado de escribir sin éxito, pero no sin dedicación, las siguientes novelas fracasadas. La novela "verdadera", la historia del asalto al camión pagador, la fuga y el encierro de cuatro maleantes en un departamento de Montevideo en el que resisten toda la noche a la policía y, antes de morir, queman el dinero. Uso del grabador y una técnica que transmite la oralidad de los personajes como un documento real. Una novela corta centrada en Pavese, que transcurre en Italia y gira sobre los diarios personales. (III, 101) 
19 Aquí, de nuevo, reflexiona sobre cuestiones de la técnica narrativa y el uso de una grabadora (o la simulación de una), para lograr el efecto de oralidad (Premat 2004: 125-127). Otra entrada sin fecha (cuando deja de lado la estructura del diario per se), dice:

Bajó por Callao, caminando por la vereda del sol, la librería en la esquina de la Universidad del Salvador, y al cruzar Córdoba, la funeraria. Una tarde en el 67, cuando vivía en un departamento en la cortada Del Carmen, había encontrado el comienzo de la novela en la que trabajaba desde hacía meses, los malandras secuestraban a un periodista, y durante el asedio él grababa sus historias en un grabador portátil, buscaba registrar los ritmos del habla, recordaba de memoria el primer párrafo, había tardado dos meses en escribir esas veinte líneas y se mantuvo fiel al tono, los personajes se alternaban en esa novela, tenía los periódicos de la época, el asalto al camión recaudador, la fuga al Uruguay, el cerco policial, la resistencia suicida hasta el final, cuando deciden quemar la plata, pero antes dejan libre al periodista para que cuente la historia. Había visto ese día, como una aparición, nítida, la imagen del hombre alto, lívido, ahogado por el humo y los gases, que salía del departamento en ruinas y cruzaba entre las cenizas y los cadáveres con el grabador en alto, como un soldado que lleva su fusil sobre la cabeza al cruzar un río. (III, 179)

Aquí está recordando una falsa salida: la novela de 1997 no tiene esta naturaleza periodística y Haberkorn no menciona ese incidente en su libro. Hay un desplazamiento fascinante de las reflexiones sobre la grabadora de Oscar Lewis y los inicios del testimonio hacia el marco de la novela, a pesar de que este marco fuera finalmente descartado (sería interesante ver si el primer párrafo, aquí indicado, está en los materiales en la biblioteca de Princeton). La última referencia a la novela está en una carta al compositor Gerardo Gandini (con quien había trabajado en la versión para ópera de La ciudad ausente, que se estrenó en el Teatro Colón en 1995), que trata de una visita que haría Gandini a Princeton. En ella, Piglia comenta sobre su estado de ánimo a causa de las demandas judiciales por Plata quemada y las demandas subsecuentes: "Estoy desanimado y furioso (extraña mezcla que algún efecto va a producir), trato de no hacerme la víctima, porque los tipos que me atacan y quieren mandarme preso cultivan la poética de la víctima, de los canallas que se hacen los mártires" (III, 227). Esta es la única alusión a la novela después de su publicación en el tercer volumen del diario.

Piglia comenta en el epílogo de Plata quemada que "Esta novela cuenta una historia real" (1997: 245)7. Explica que ha tratado de ser fiel a la manera de hablar de los protagonistas y los testigos (eco de la entrada sobre Oscar Lewis en el diario, fechada en diciembre de 1967) y que usó entrevistas publicadas en el periódico El Mundo de Buenos Aires (y aquí Piglia está jugando con el lector, porque ése fue el periódico para el cual Roberto Arlt escribió muchos años antes), entrevistas con Dorda, con varios jueces y testigos en Montevideo, un abogado, un policía y varios otros testigos en Buenos Aires, así como artículos publicados en varios periódicos: Crónica, Clarín, La Nación y La Razón, de Buenos Aires, y El Día, Acción, El País y Debate de Montevideo (248). Le hace un guiño al lector con la declaración: "Fueron de especial utilidad las crónicas y las notas de quien firmaba E. R., que cubrió el asalto y fue el enviado especial del diario argentino El Mundo al lugar de los hechos" (248), ya que Emilio Renzi es alter ego y "autor" de Los diarios de Emilio Renzi, nombre derivado del suyo propio, Ricardo Emilio Piglia Renzi. Comenta también que, en un tren hacia Bolivia en marzo o abril de 1966, tuvo una larga conversación con Blanca Galeano - "la Nena" en la novela-, quien había tenido una relación amorosa con el "pistolero Mereles" (249) y quien le dio "una primera y confusa versión de los hechos que yo recordaba vagamente haber leído en los diarios meses atrás" (249-250). Añade que: 
Después la chica siguió sola el viaje a La Paz y nunca más la vi. Recuerdo que en el tren y en la estación y luego en el hotel tomé algunas notas de lo que me contó (porque en aquel tiempo yo consideraba que un escritor debía ir a todos lados con una libreta de notas) y poco después (en 1968 o 1969) inicié la investigación y escribí una primera versión de este libro.

Siempre serán misteriosas para mí las razones por las que algunas historias se resisten durante años a ser contadas y exigen un tiempo propio. Abandoné el proyecto en 1970 y mandé los borradores y los materiales a la casa de mi hermano. Hace un tiempo, en medio de una mudanza, encontré la caja con los manuscritos y los documentos en los que estaban los resultados principales de la investigación y la primera redacción del libro. En el verano de 1995 comencé a escribir de nuevo por completo la novela, tratando de ser absolutamente fiel a la verdad de los hechos. Los acontecimientos estaban ahora tan distantes y tan cerrados, que parecían el recuerdo perdido de una experiencia vivida. Casi los había olvidado ya y eran nuevos y casi desconocidos para mí luego de más de treinta años. Esa lejanía me ha ayudado a trabajar la historia como si se tratara del relato de un sueño. (250-251)

El epílogo de 1997 - que, como algunas notas del diario, compara la historia oída con una tragedia griega ("yo la escuché como si me encontrara frente a una versión argentina de una tragedia griega" [250]) - cambia levemente la cronología del proyecto y sitúa las investigaciones y la escritura en 1968 y 1969. El diario sugiere, en cambio, que el periodo clave fue de enero de 1966 a diciembre de 1967, pero hasta poder consultar plenamente los vastos materiales que están en Princeton es imposible establecer con certeza qué se escribió en la década del 60 y qué fue reescrito bajo circunstancias tan complejas que son difíciles de imaginar, en los varios años entre el diagnóstico de la ELA de Piglia en 2013-2014 y su muerte en enero de 2017. Lo que impresiona al lector es, sin embargo, la consistencia entre las entradas del diario y las aseveraciones sobre el proceso de escribir que están en el epílogo de 1997: tal parece que el epílogo a la novela, como la novela misma, "cuenta una historia real" (245). El género de la no ficción, el nuevo modo de escribir cuyas raíces encontramos en Oscar Lewis (y la ejecución de tal modo por el argentino Rodolfo Walsh en Operación masacre [1957], que se considera un antecedente importante), que fue cultivado en los años sesenta por Truman Capote, Norman Mailer, Hunter Thompson y Tom Wolfe, son determinantes en las primeras etapas del proyecto. La novela de Piglia es un homenaje a ese género, a pesar de que el producto final cambió bastante respecto a lo sugerido en las entradas del diario. Como escribe Piglia en su introducción de 1967 a Capote, recogida en Escritores norteamericanos, "fiel a sí mismo, Capote ha revolucionado la novela moderna, ha inaugurado la non-fiction pero, sobre todo, ha rescatado lo mejor del universo de sus primeras narraciones: lo ha endurecido y concentrado, pero sin traicionarlo" (2016: 49). Al tiempo que escribe estas palabras sobre Capote, está compilando los materiales que treinta años después se transformarían en Plata quemada; su novela de no-ficción se alejará del modelo, pero, asunto interesante, desarrollará la línea homoerótica tan importante en Capote, no sólo en una novela de tema explícitamente homosexual como Other Voices, Other Rooms, sino también en las tensiones subyacentes de In Cold Blood. La novela de Piglia, un lúcido replanteamiento del género, explora cómo una historia real se puede volver a contar oblicuamente, con sutiles cambios en la focalización que socavan la certeza de las versiones de las historias. Como anticipó en las entradas del diario de 1966 a 1967, el problema central en la novela -que en el diario fue llamada sucesivamente La novela, El robo y Entre hombres, y Plata quemada en la posterior publicación- será cómo contar la historia. Es decir, la pregunta central es la misma que plantea el narrador a comienzos de Respiración artificial de 1980: “¿Hay una historia? Si hay una historia empieza hace tres años" (13). 


\section{BIBLIOGRAFÍA}

Balderston Daniel, "El Borges de Piglia”, Teresa Orecchia Havas (ed.), Homenaje a Ricardo Piglia, Buenos Aires, Catálogos, 2012, p. 145-155.

Clayton Michelle, “Cómo habla la plata”, Adriana Rodríguez Pérsico (ed.), Ricardo Piglia: una poética sin límites, Pittsburgh, Instituto Internacional de Literatura Iberoamericana, 2004, p. 135-144.

Haberkorn Leonardo, Liberaij: La verdadera historia del caso Plata quemada, Montevideo, Sudamericana Uruguaya, 2015.

--- “Liberaij: La verdadadera historia del caso Plata Quemada: Entrevistas, reseñas, críticas.” Blog. Web.

Larre Borges Ana Inés, “Un delito que prescribe”, Brecha, 7 de agosto de 2015, p. 22-23.

Piglia Ricardo, Respiración artificial, Buenos Aires, Pomaire, 1980.

--- Plata quemada, Buenos Aires, Planeta, 1997.

--- Los diarios de Emilio Renzi: Años de formación, Barcelona, Anagrama, 2015.

--- Los diarios de Emilio Renzi: Los años felices, Barcelona, Anagrama, 2016.

--- Escritores norteamericanos, Buenos Aires, Tenemos Las Máquinas, 2016.

--- Los diarios de Emilio Renzi: Un día en la vida, Barcelona, Anagrama, 2017.

Premat Julio, “Los espejos y la cópula son abominables”, Adriana Rodríguez Pérsico (ed.), Ricardo Piglia: una poética sin límites, Pittsburgh, Instituto Internacional de Literatura Iberoamericana, 2004, p. 123-134.

Rodríguez Pérsico Adriana, "Plata quemada o un mito para el policial argentino", Ricardo Piglia: una poética sin límites, Pittsburgh, Instituto Internacional de Literatura Iberoamericana, 2004, p.

113-121.

\section{NOTAS}

1. Agradecemos la traducción de este trabajo a la amabilidad de Edgar Luis Colón (Nota de la editora del volumen. Todas las notas que siguen a ésta pertenecen al autor del artículo).

2. Esto difiere de lo expresado por Haberkorn.

3. Por razones de simplicidad, en adelante me referiré a los Los diarios de Emilio Renzi por volumen: I será Los años de formación (2015), II Los años felices (2016) y III Un día en la vida (2017).

4. Debo subrayar que Piglia y sus asistentes seleccionaron, y tal vez reescribieron, porciones del diario; pude consultar parte de los diarios que están ahora en la biblioteca de Princeton en abril de 2018, pero en este artículo el diario publicado será la principal fuente de investigación. No puedo dar fe de que esta cita corresponde con exactitud a lo escrito por Piglia en noviembre de 1965.

5. Es interesante que usa la palabra de argot "malandra", asociada a un término clave que Antonio Candido usó en un famoso ensayo titulado "Dialética da malandragem" (publicado poco 
después, en los años setenta) sobre Memórias de um sargento de milícias de Manuel Antonio de Almeida.

6. Rodríguez Pérsico cita las ideas de Piglia sobre las relaciones homosexuales, o lo que prefiere llamar "circulaciones del deseo, que se dan entre hombres a veces y se dan entre hombres y mujeres o entre mujeres". Añade: "Me parece que en mundo popular, en las clases bajas, este juego de las identidades sexuales, como quiera que sean [,] son menos fijas" (2004: 116 n3).

7. Premat reflexiona sobre esta frase y sus implicaciones para la novela (2004: 124), y sobre el epílogo (127-130).

\section{RESÚMENES}

Este artículo examina Los diarios de Emilio Renzi a fin de informar sobre la investigación que realizó Ricardo Piglia a finales de los años sesenta para una novela, Plata quemada, que no se publicaría hasta 1997.

L'article examine Los diarios de Emilio Renzi dans le but de recueillir information sur les recherches faites par Ricardo Piglia à la fin des années soixante,pour son roman Plata quemada, qui ne serait publié qu'en 1997.

This paper examines Los diarios de Emilio Renzi for information about Ricardo Piglia's research in the late 1960s for the novel Plata quemada that he wouldn't publish until 1997.

\section{ÍNDICE}

Mots-clés: Ricardo Piglia, vol de San Fernando, fusillade de l'immeuble Liberaij, journaux, Plata quemada.

Keywords: Ricardo Piglia, San Fernando robbery, Liberaij shootout, diaries, Plata quemada Palabras claves: Ricardo Piglia, asalto de San Fernando, tiroteo del Edificio Liberaij, diarios, Plata quemada

\section{AUTOR}

\section{DANIEL BALDERSTON}

University of Pittsburgh

dbalder@pitt.edu 